Classification

Physics Abstracts

$05.40-61.40 \mathrm{~K}-64.70$

\title{
Asymptotic behaviour of the form factor of a long polymer in a good solvent
}

\author{
J. des Cloizeaux and B. Duplantier
}

Service de Physique Théorique, CEN Saclay, 91191 Gif sur Yvette Cedex, France

(Reçu le 18 février 1985, révisé le 27 mars, accepté le 28 mars 1985)

Résumé. - Le facteur de forme d'un long polymère en bon solvant peut s'écrire sous forme de loi d'échelle $H(q)=h\left(q^{2} R^{2} / 2 d\right)($ avec $h(0)=1)$ où q est le vecteur de transfert, $d$ la dimension de l'espace et $R^{2}$ la distance carrée moyenne bout à bout. Pour de grandes valeurs de $x, h(x) \simeq \frac{h_{\infty}}{x^{1 / 2 v}}$ où $v \simeq \frac{1}{2}\left(1+\frac{\varepsilon}{8}+\cdots\right)$ et par renormalisation directe, on trouve $h_{\infty} \simeq 2\left[1-\frac{\varepsilon}{8}(5-C)+\cdots\right]$ où $C \simeq 0,577$ (constante d'Euler).

Abstract. - The form factor of a long polymer in a good solvent can be written in the form of a scaling law $H(q)=h\left(q^{2} R^{2} / 2 d\right)$ (with $h(0)=1$ ) where $q$ is the transfer vector, $d$ the space dimension and $R^{2}$ the mean square end to end distance. For large values of $x, h(x) \simeq \frac{h_{\infty}}{x^{1 / 2 v}}$ where $v \simeq \frac{1}{2} \times$ $\left(1+\frac{\varepsilon}{8}+\cdots\right)$ and by direct renormalization we find $h_{\infty} \simeq 2\left[1-\frac{\varepsilon}{8}(5-C)+\cdots\right]$ where $C \simeq 0.577$ (Euler's constant).

\section{Introduction.}

Our aim is to study the asymptotic properties of the form factor of a long polymer in a good solvent. The form factor $H(q)$ can be defined in the framework of the continuous model $[1,2]$ by :

$$
H(\mathbf{q})=\frac{1}{S^{2}} \int_{0}^{S} \mathrm{~d} s^{\prime} \int_{0}^{S} \mathrm{~d} s^{\prime \prime}\left\langle\mathrm{e}^{i \mathbf{q} \cdot\left[\mathbf{r}\left(s^{\prime}\right)-\mathbf{r}\left(s^{\prime \prime}\right)\right]}\right\rangle
$$

where $\mathbf{r}(s)$ defines the curve representing the polymer. Here $s$ is a parameter which is proportional to the number of links; it can be considered as a "Brownian area " [2]. We may also define the size $X$ of the polymer by setting :

$$
\left\langle[\mathbf{r}(s)-\mathbf{r}(0)]^{2}\right\rangle=d X^{2}
$$

where $d$ is the dimensionality of the space in which the polymer is immersed. 
The form factor of a long polymer in a good solvent is given by a scaling law of the form :

$$
H(\mathbf{q})=h\left(q^{2} X^{2} / 2\right) .
$$

The function $h(x)$ for small $x$ has been calculated by Witten and Schäfer [3] (see also [4]) who found (in our notation) :

$$
h(x)=1-\frac{x}{3}\left(1-\frac{\varepsilon}{96}\right)+\frac{x^{2}}{12}\left(1-\frac{19 \varepsilon}{240}\right) .
$$

Here we consider the limit $x=q^{2} X^{2} / 2 \gg 1$. In this limit, the chain may be considered as infinite and $H(\mathbf{q})$ may be defined by the approximate expression :

$$
H(\mathbf{q})=\frac{2}{S} \int_{0}^{\infty} \mathrm{d} s\left\langle\mathrm{e}^{i \mathbf{q} \cdot[\mathbf{r}(s)-\mathbf{r}(0)]}\right\rangle
$$

We see that in this limit $H(q)$ must be inversely proportional to the number of links which, in the asymptotic regime, is measured by $X^{1 / v}$. Thus for large $x$, we have :

$$
h(x) \simeq \frac{h_{\infty}}{x^{1 / 2 v}} \quad\left(H(q) \propto \frac{1}{q^{1 / v}}\right) .
$$

In particular for a Brownian chain :

$$
\begin{aligned}
{ }^{0} h(x) & =\frac{2}{x^{2}}\left(\mathrm{e}^{-x}-1+x\right) \quad \text { (Debye's formula) } \\
{ }^{0} h_{\infty} & =2 .
\end{aligned}
$$

Here, we shall calculate $h_{\infty}$ to order $\varepsilon=4-d$ (where $d$ is the space dimension) by using the direct renormalization method [2]. The calculation of a quantity similar to $h_{\infty}$ has also been performed by T. Witten [5], who used the grand canonical ensemble and a field theoretical method $\left(^{1}\right)$.

\section{Calculation.}

For large $\mathbf{q}, H(\mathbf{q})$ is given by :

$$
H(\mathbf{q})=\frac{2}{S} \int_{0}^{\infty} \mathrm{d} s \frac{3(\mathbf{q}, s)}{3(0, s)}
$$

where $3(\mathbf{q}, s)$ is a partition function with insertions of wave vector $\mathbf{q}$ and $-\mathbf{q}$ at two points separated by a Brownian area $s$ along the infinite chain. The contributions of orders zero and one, with respect to the interaction $b$, correspond to the diagrams represented on the figure.

( ${ }^{1}$ ) After the completion of our work, reference [5] was pointed out to us by one of the referees, whom we wish to thank. Our calculation is different and much simpler. 


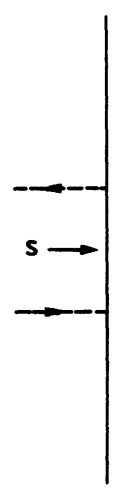

(a)

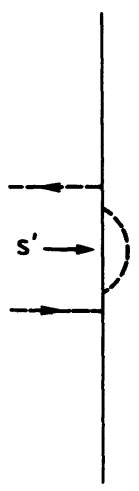

(b)

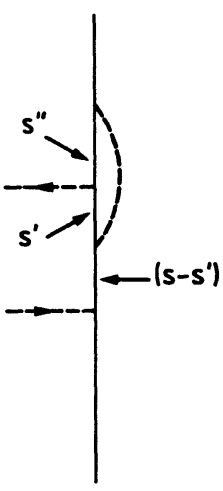

(c)

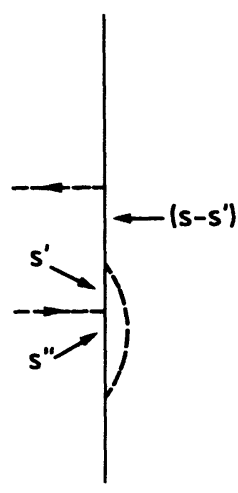

(d)

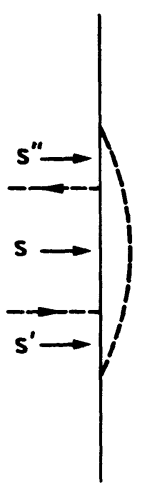

(e)

Fig. 1. - Diagrams contributing to $H(\mathbf{q})$ for large values of q. (a) Diagram of order zero. (b-e) Diagrams of order one. In order to get Eq. (4), we set : $t=q^{2} \frac{s}{2} ; s^{\prime}=t^{\prime} s ; s^{\prime \prime}=t^{\prime \prime} s$ where $s, s^{\prime}, s^{\prime \prime}$ are « areas " of polymer segments.

Using the notation :

$$
\begin{aligned}
& z=b S^{2-d / 2}(2 \pi)^{-d / 2} \\
& y=q^{2} S / 2
\end{aligned}
$$

we obtain to first order in $z$ :

$$
H(\mathbf{q})=\frac{2}{y}-\frac{2 z}{y^{3-d / 2}}\left[I_{\mathrm{b}}+I_{\mathrm{c}}+I_{\mathrm{d}}+I_{\mathrm{e}}\right]
$$

where $I_{\mathrm{b}}, I_{\mathrm{c}}, I_{\mathrm{d}}$ and $I_{\mathrm{e}}$ are the contributions of the diagrams shown on the figure. We have :

$$
\begin{aligned}
& I_{\mathrm{b}}=\int_{0}^{\infty} \mathrm{d} t \int_{0}^{t} \mathrm{~d} t^{\prime}\left(t-t^{\prime}\right) \frac{\left[\mathrm{e}^{-\left(t-t^{\prime}\right)}-\mathrm{e}^{-t}\right]}{t^{\prime d / 2}}=\frac{2}{\varepsilon}+1-C+\cdots \\
& I_{\mathrm{c}}=I_{\mathrm{d}}=\int_{0}^{\infty} \mathrm{d} t \int_{0}^{t} \mathrm{~d} t^{\prime} \int_{0}^{\infty} \mathrm{d} t^{\prime \prime} \frac{\left\{\exp \left[-\left(t-\frac{t^{\prime 2}}{t^{\prime}+t^{\prime \prime}}\right)\right]-\mathrm{e}^{-t}\right\}}{\left(t^{\prime}+t^{\prime \prime}\right)^{d / 2}}=1+\cdots \\
& I_{\mathrm{e}}=\int_{0}^{\infty} \mathrm{d} t \int_{0}^{\infty} \mathrm{d} t^{\prime} \int_{0}^{\infty} \mathrm{d} t^{\prime \prime} \frac{\left\{\exp \left[-\left(t-\frac{t^{2}}{t+t^{\prime}+t^{\prime \prime}}\right)\right]-\mathrm{e}^{-t}\right\}}{\left(t+t^{\prime}+t^{\prime \prime}\right)^{d / 2}}=1+\cdots
\end{aligned}
$$


Thus, we find :

$$
H(\mathbf{q})=\frac{2}{y}-\frac{2 z}{y^{1+\varepsilon / 2}}\left(\frac{2}{\varepsilon}+4-C\right)
$$

where $C$ is the Euler constant $(C \simeq 0.577)$.

Let us now express $h(\mathbf{q})$ in terms of $x$ by introducing the swelling factor $\mathfrak{X}_{0}(z)$. Using the previous results [1], we may write :

$$
\frac{x}{y}=\frac{X^{2}}{S} \equiv X_{0}(z) \simeq 1+\frac{z}{\varepsilon}(2-\varepsilon)
$$

and we obtain in this way :

$$
H(\mathbf{q})=\frac{2}{x}+\frac{2 z}{x}(C-5+\ln x) .
$$

Now it is possible to express $z$ in terms of the dimensionless parameter $g$ which defines the second virial coefficient of a solution of long polymers [2].

We have according to previous results [2] :

$$
z=g+\cdots
$$

and in equation (6), we may replace $z$ by $g$.

Moreover, we know that, when $z \rightarrow \infty$, then :

$$
g \rightarrow g^{*}=\frac{\varepsilon}{8} .
$$

In this limit, we obtain :

$$
H(\mathbf{q}) \simeq h(x)=\frac{2}{x}+\frac{\varepsilon}{4 x}(C-5+\ln x) .
$$

We may also write this expression in the form :

$$
h(x)=\frac{2\left[1-\frac{\varepsilon}{8}(5-C)\right]}{x^{1-\varepsilon / 8}}
$$

which is compatible with equation (3) since :

$$
v=\frac{1}{2}\left(1+\frac{\varepsilon}{8}+\cdots\right)
$$

Finally, by comparing equations (3) and (7), we find the final result :

$$
h_{\infty}=2\left(1-\frac{\varepsilon}{8}(5-C)+\cdots\right) \text {. }
$$

Thus, for $\varepsilon=1(d=3)$, we get the approximate value $h_{\infty}=0.89$. The value obtained in reference [5] is not written in this form but, after some transformations, is found to agree with (8). 
Incidentally, we note that, using simple assumptions, Peterlin [6] (1955), Benoit [7] (1957) and Ptitsyn [8] (1957) have derived an approximate expression of $H(\mathbf{q})$ which is a simple generalization of Debye's formula.

The corresponding value of $h(x)$ is :

$$
h_{\mathrm{A}}(x)=2 \int_{0}^{1} \mathrm{~d} t(1-t) \mathrm{e}^{-x t^{2 v}}
$$

and for $v=\frac{1}{2}(1+\varepsilon / 8)$ this gives for $0<x \ll 1$ :

$$
h_{\mathrm{A}}(x)=1-\frac{x}{3}\left(1-\frac{5 \varepsilon}{48}\right)+\frac{x^{2}}{12}\left(1-\frac{7 \varepsilon}{48}\right)
$$

and for $x \gg 1$ :

$$
h_{\mathrm{A}}(x)=\frac{2}{x^{1-\varepsilon / 8}}\left[1-\frac{\varepsilon}{8}(1-C)\right]
$$

Comparing equations (10) and (11) to the « exact » results given by (2) and (7), we note a great discrepancy (in particular for $\varepsilon=1$, Eq. (11) would give $h_{\infty}=1.89$ ). This shows that equation (9) cannot be trusted, a fact which is not unexpected since (9) relies on fairly crude assumptions. In (9) the swelling of all parts of the chain is assumed to be given by the same law as that of the ends of the chain. But the swelling of the internal parts of the real chain is actually larger (though with the same index). This can be seen on the radius of gyration, a quantity which is sensitive to the internal parts of the chain. Using equation (2), we find :

$$
R_{\mathrm{G}}^{2}=d X^{2}\left(1-\frac{\varepsilon}{96}\right)
$$

while (10) implies

$$
R_{\mathbf{G}}^{2 \prime}=d X^{2}\left(1-\frac{5}{48} \varepsilon\right)
$$

a value which is smaller because it underestimates the contributions of the internal parts. A similar argument explains why our value of $h_{\infty}$ is smaller than the value given by (11).

\section{References}

[1] Yamakawa, H., Modern Theory of Polymer Solutions (Harper and Row) 1971.

[2] Des Cloizeaux, J., J. Physique 42 (1981) 635.

[3] Witten, T. and SchäFER, L., J. Chem. Phys. 74 (1981) 2582.

[4] Ohta, T., OONo, Y., FreED, K., Macromolecules 14 (1981) 1588. The result of [3] can be represented by equation (2) and agrees with the corresponding result of [4], in spite of a statement contained in [4].

[5] Witten, T., J. Chem. Phys. 76 (1982) 3300.

[6] Peterlin, A. J., J. Chem. Phys. 23 (1955) 2464.

[7] Benoit, H., C.R. Hebd. Séan. Acad. Sci. Paris 245 (1957) 2244.

[8] PTitsyn, O. B., Zh. Fiz. Khim 31 (1957) 1091. 